MUZIKOLOŠKI ZBORNIK • MUSICOLOGICAL ANNUAL XL

UDK 78(497.5:4-014)

Stanislav Tuksar

Universität Zagreb

Univerza v Zagrebu

\title{
Kroatische Kunstmusik und ihr Verhältnis zum mitteleuropäischen Raum
}

\section{Hrvaška umetna glasba in njen odnos do srednjeevropskega prostora}

\section{ZUSAMMENFASSUNG}

Die Formulierung dieses Themas - Kroatische Kunstmusik und ihr Verhältnis zum mitteleuropäischen Raum - regt bereits zu einer fundamentalen Diskussion an. Es scheint, nämlich, daß hier zwei Entitäten enthalten sind, die ihrem Wesen nach voneinander abgetrennt sind und als solche in einem übergeordneten Kontext, bzw. im Begriff und in der Realität der europäischen Musik und ihrer geographisch bestimmten Geschichte koexistieren, sodaß deren konkrete Erscheinungsformen und Relationen verschiedene Formen und Modalitäten annehmen können. In weiterer Diskussion sollen vorläufig einige Determinanten der beiden Begriffe (kroatische Kunstmusik; mitteleuropäischer Raum) dargestellt werden und eine der möglichen Lösungen vorgestellt sein.

Kroatische Kunstmusik ist zu verstehen als die Gesamtheit der kunstmusikalischen Produktion und ihres geistigen und sozialen Zusammenhangs, die auf Grund der Wirkung vieler Komponisten, Interpreten und Musikforscher zustandegekommen sind; unter diesen hauptsächlich aus Kroatien abstammenden Komponisten, Interpreten und Musikforschern sind auch solche zu finden, die, von ihrer ausländischen Herkunft abgesehen, ihre Tätigkeit in einem respektablen Umfang innerhalb der Kulturkreise in historisch kroatischen Ländern (Slawonien, engeres Kroatien, Dalmatien mit Dubrovnik und zum Teil Istrien) ausgeübt hatten, wobei
POVZETEK

Sama artikulacija te teme - hrvaška umetna glasba in njen odnos do srednjeevropskega prostora odpira temeljno diskusijo. Zdi se namreč, da gre za dve entiteti - ki sta $\mathrm{v}$ bistvu ločeni in kot taki obstajata $\mathrm{v}$ nekem nadkontekstu oziroma kot pojem in realnost evropske glasbe in njene geozgodovine - katerih konkretna pojavnost in odnosi lahko dobijo različne oblike in modalitete. $\mathrm{V}$ nadaljnji razpravi so predstavljena nekatera določila enega in drugega pojma (hrvaška umetna glasba, srednjeevropski prostor), zaključek pa pripelje do ene izmed možnih rešitev.

Hrvaška umetna glasba je razumljena kot spoj glasbenoumetnostne produkcije in njenega duhovno-družbenega konteksta. Slednjega so ustvarili skladatelji, izvajalci in misleci o glasbi, ki so po rodu Hrvati oziroma tisti Nehrvati, ki so svoje delovanje $\mathrm{v}$ pomembnem obsegu opravili znotraj kulturnih krogov hrvaških zgodovinskih dežel - Slavonije, ožje Hrvaške, Dalmacije z Dubrovnikom in delo$\mathrm{ma}$ Istre - in to $\mathrm{v}$ dokumentiranem in zato dokazanem kontinuiranem trajanju najmanj deset stoletij. Ta skupek glasbenokulturnih dejstev kaže od svojih kronoloških začetkov karakteristike, ki ga vežejo ob bok strukturam, oblikam in funkcijam umetne glasbe evropskega Zahoda. To spoznanje se potrjuje prav tako na pojavih cerkvene glasbe od 10 . stoletja naprej, povezane primarno $z$ liturgično-verskim kontekstom zahodne glasbe katoliškega kro- 


\section{MUZIKOLOŠKI ZBORNIK • MUSICOLOGICAL ANNUAL XL}

es um eine dokumentierte und daher auch nachgewiesene, mindestens zehn Jahrhunderte umfassende Kontinuität geht. Diese Gesamtheit musikalisch-kultureller Fakten weist bereits seit ihren chronologisch erfaßten Anfängen Charakteristiken auf, durch die sie mit Strukturen, Formen und Funktionen der Kunstmusik des europäischen Westens engstens verbunden ist. Diese Erkentniss wurde schon seit dem 10. Jh. an der Kirchenmusik verifiziert, die stets mit dem liturgisch-sakralen Kontext der westlichen Musik - größtenteils katholischer Provenienz - verknüpft war (zusammen mit sekundärer Musikerbe einiger Ostkirchen, der jüdischen Gemeinden usw.). Ebenso erfüllte die profane kroatische Kunstmusik - im Hinblick auf vokale, vokalinstrumentale und instrumentale Genres und Gattungen - ihre individualpsychologische und soziale Funktionen nach entsprechenden westlichen Modellen seit der Renaissancezeit bis zum 20. Jh.

Der mitteleuropäische Raum auf dem Gebiet der Kultur und Musik bleibt nach wie vor als Kulturbegriff und die bestimmte räumliche Entität schwer zu definieren, wenngleich viele intuitiv zu ahnen und empirisch zu spüren geneigt sind, daß es sich hier nicht um eine reine Fiktion handelt, sondern um einen Umfeld der Gravitationspunkte in der geistigen Geographie Westeuropas, soziologisch und kulturologisch bestimmbar als deren Übergangsgebiet Osteuropa gegenüber. In Kroatien ist dieser Raum allenfalls plurizentrisch $\mathrm{zu}$ verstehen - Graz, Salzburg, Wien und Österreich, Budapest, Pécs und Ungarn, Mannheim, Dresden, München und Süddeutschland, Prag, Brünn und Tschechien, Bratislava, Trnava und der Slowakei, Warschau, Krakau und Polen, Ljubljana, Maribor und Slowenien, Zagreb und Nordwestkroatien umfassend, inklusive einige norditalienische Provinzen wie Veneto (ohne Venedig) und Furlanien mit Udine und Triest.

Es wurde festgestellt, dass das zentrale Problem hinsichtlich der Identität der kroatischen Musikkultur im engeren und der allgemeinen Kultur im weiteren Sinn des Wortes keine künstliche Frage ist die sich durch die Logik der sogenannten hohen Politik, der Medien und der Linguistik in den letzten Jahrhundert durchgesetzt hat - ob Kroatien zu Mitteleuropa oder zum europäischen Osten gehörig sei, sondern dass es sich um eine geistige Geometrie handelt, wo sich die Einflußsphären des musikalischen Nordens (Mitteleuropa) und Südens (Mediterran) als osmotische Abgrenzung überlappen.

Darum wird die Antwort auf die fundamentale Frage nach dem Verhältnis der kroatischen Kunstmusik zum mitteleuropäischen Raum lauten: die kroatische Kunstmusik und ihre Musik- ga (ob sekundarni glasbeni dediščini nekaterih vzhodnih cerkvenih obredov, židovski glasbi itd.) kot tudi posvetne glasbe od 16. stoletja naprej, katere oblike in družbene funkcije sledijo modelom in obrazcem zahodnih kulturnih krogov v vokalnih, vokalno-instrumentalnih in instrumentalnih žanrih in vrstah od renesanse do 20 . stoletja.

Srednjeevropski prostor je na področju kulture in glasbe težko določiti kot definirano prostorsko entiteto, vendar se intuitivno sluti in empirično čuti, ne kot gola fikcija, ampak kot skupek gravitacijskih točk v duhovni geografiji Zahodne Evrope določenih pojmovno, sociološko in kulturološko kot njeno prehodno področje proti vzhodu Evrope - na Hrvaškem pa vsekakor kot večsrediščni prostor, ki vključuje središča in države kot npr. Gradec, Salzburg, Dunaj in Avstrija; Budimpešta, Pécs in Madžarska; Mannheim, Dresden, München in južna Nemčija; Praga, Brno in Češka; Bratislava, Trnava in Slovaška; Varšava, Krakov in Poljska; Ljubljana, Maribor in Slovenija; Zagreb in severozahodna Hrvaška in deloma tudi severnoitalijanske pokrajine Benečije (brez Benetk) in Furlanije z Vidmom in Trstom.

Tako se potrjuje, da osrednji problem identitete spoja hrvaške glasbene in splošne kulture ni umetno vprašanje, postavljeno z logiko t. i. visoke politike, medijev in lingvistike $\mathrm{v}$ zadnjih sto letih o Hrvaški kot državi in kulturi v Srednji Evropi ali na evropskem Vzhodu, ampak o področju, na katerem se $v$ duhovni geometriji v obliki osmozne razmejitve menjajo vplivne sfere njegovega glasbenega severa (Srednja Evropa) in juga (Mediteran).

Zato se na temeljno vprašanje o odnosu hrvaške umetne glasbe in srednjeevropskega prostora odgovarja: hrvaška umetna glasba in glasbena kultura niso monolitne. Po svojem značaju in po svojem pomembnem delu so del srednjeevropskega prostora z vgrajeno močno mediteransko komponento. Pri tem ne gre pozabiti dejstva, da je Hrvaška na robu Srednje in na drugi strani Zahodne Evrope ter da je Hrvaška prehodna država in kultura proti njenem vzhodu, vendar da je nedvomno država zahodnoslovanskega in s tem tudi srednjeevropskega glasbenokulturnega kroga. 
MUZIKOLOŠKI ZBORNIK • MUSICOLOGICAL ANNUAL XL

kultur sind keineswegs in sich einheitlich zu sehen und lassen sich in einem gewichtigen Teil als ein Bestandteil des mitteleuropäischen Raumes wahrnehmen, aber mit eine eingebaute wichtige mediterrane Komponente. Dabei ist immer klar zu sehen, dass Kroatien sich am Rande des mittleren und darüber hinaus des westlichen Europas befindet und dass es ein Übergangsland und eine Übergangskultur in Richtung Osten ist, aber dass auch Kroatien unbestrittenerweise auch ein Land aus dem westslawischen und dadurch mitteleuropäischen musikalisch-kulturellen Umkreis ist.

Das Thema des vorliegenden Beitrags - Kroatische Kunstmusik und ihr Verhältnis zum mitteleuropäischen Raum - regt bereits zu einer fundamentalen Diskussion an. Es entsteht nämlich der Eindruck, daß in der so artikulierten Schwerpunktformulierung zwei Entitäten enthalten sind, die ihrem Wesen nach voneinander abgetrennt sind und als solche in einem übergeordneten Zusammenhang bzw. im Begriff und in der Realität der europäischen Musik und ihrer geographisch bestimmten Geschichte koexistieren, sodaß deren konkrete Erscheinungsformen und Relationen verschiedene Formen und Modalitäten annehmen können. Um in dieser Richtung auch weiterhin - sei es selbst auf der grundlegenden Ebene - überhaupt noch diskutieren zu können, sollen vorläufig einige Determinanten der beiden Begriffe (kroatische Kunstmusik; mitteleuropäischer Raum) dargestellt werden.

Was bedeutet also das Syntagma "kroatische Kunstmusik»? Es sollte meines Erachtens keinen Zweifel darüber geben, daß es sich hier um die Gesamtheit der kunstmusikalischen Produktion und ihres geistigen und sozialen Zusammenhangs handelt, die auf Grund der Wirkung vieler Komponisten, Interpreten und Musikforscher zustandegekommen sind; unter diesen hauptsächlich aus Kroatien abstammenden Komponisten, Interpreten und Musikforschern sind auch solche zu finden, die, von ihrer ausländischen Herkunft abgesehen, ihre Tätigkeit in einem respektablen Umfang innerhalb der Kulturkreise in historisch kroatischen Ländern (Slawonien, engeres Kroatien, Dalmatien mit Dubrovnik und zum Teil Istrien) ausgeübt hatten, wobei es um eine dokumentierte und daher auch nachgewiesene, mindestens zehn Jahrhunderte umfassende Kontinuität geht. Diese Gesamtheit musikalisch-kultureller Fakten weist bereits seit ihren chronologisch erfaßten Anfängen Charakteristiken auf, durch die sie mit Strukturen, Formen und Funktionen der Kunstmusik des europäischen Westens engstens verbunden ist.

Die ganze Sphäre der kroatischen Kirchenmusik ist mit dem liturgisch-sakralen Kontext der westlichen Musik - größtenteils katholischer Provenienz - verknüpft. Die erhalten gebliebenen mittelalterlichen Kodizes und deren Fragmente sowie die später entstandenen Choralbücher bringen das Repetoire und die Schreibweisen zum Vorschein, die mit dem beneventanischen Komplex und/oder mit den in kroatischen Landen bereits gepflegten bzw. aus benachbarten Diözesen Aquileia, Salzburg, Kalocsa und anderen Mittelpunkten aus Österreich, Ungarn, Süddeutschland und 
Italien nachträglich übernommenen Richtlinien identisch oder ähnlich sind. Wenngleich es als ein nationales Spezifikum von Belang ist, bleibt die glagolitische Tradition in ihrer musikalischen Dimension im Vergleich zur vorherrschenden römisch-lateinischen Strömung eher eine Episode. Ebenfalls marginal sind Erscheinungen wie der Protestantismus, der keinen stärkeren Einfluß hinterlassen hatte (beispielsweise Mekinićs Gesangbücher ohne Noten; die Spliter Episode des Bischofs Dominis) sowie die Musik der Ostkirchen in Kroatien, die lediglich in äußerst schwer auffindbaren byzantinischen Spuren der ältesten Schicht des glagolitischen Gesangs und zum Teil in der Gesangspraxis der erst seit ungefähr drei Jahrhunderten in Kroatien lebenden griechisch-katholischen und orthodoxen Minderheit feststellbar sind. Hinzuzufügen ist hier das noch nicht erforschte jüdische Musikerbe $z$. B. in Zagreb, Dubrovnik, Osijek usw. Es erübrigt sich fast zu erwähnen, daß die kroatische katholische Kirchenmusik zur Aufführung in Kirchen bestimmt war und als solche in Kirchen zu hören war, die verschiedene Baustile der Vorromanik (beispielsweise St. Donatus in Zadar), der Romanik und Gotik (St. Anastasia in Zadar, der alte Dom zu Zagreb), der Renaissance (der Dom zu Šibenik), des Barock (Jesuitenkirchen in Dubrovnik, Zagreb, Lepoglava, Varaždin usw.) und spätere Stile als deren natürliches, kulturelles und akustisches Ambiente repräsentieren. Es ist verständlich, daß die Genres der kroatischen Kirchenmusik mit Gattungen im christlichen Westen identisch sind: gregorianisches Repertoire, Messen, Motetten, Litaneien, Responsorien, Hymnen, Oratorien usw. Ein identisches Bild ergibt auch das auf dem Gebiet der kroatischen Länder erhaltene und aufgeführte internationale Repertoire. Falls es ins Ausland gebracht wurde, befindet sich dieses musikalische Erbgut in elitären europäischen Bibliotheken (im Vatikan, in Oxford, London, Wien); ansonsten wird all das entweder in einheimischen sakralen und profanen Museen oder in Archiven und Sammlungen von Klöstern und Kirchen auf mehr als 200 Lokationen in ganz Kroatien aufbewahrt. Die diesbezüglichen Artefakte belaufen sich auf mehrere Zehntausend Notenexemplare.

Die Sphäre der profanen kroatischen Kunstmusik kann mittels entsprechender Dokumente ab dem 16. Jahrhundert nachgewiesen werden. Auch ihre Formen und deren soziale Funktionen folgen den entsprechenden Modellen und Gepflogenheiten westlicher Kulturkreise im Hinblick auf vokale, vokalinstrumentale und instrumentale Genres und Gattungen: die Produktion der Renaissance kennt die FrottolaBearbeitungen und das Ricercare (F. Bossinensis, A. Antico), Madrigale und Gregheschen (A. Petris, J. Skjavetić, L. Courtoys der Ältere); die barocke Musik artikulirt sich in Sonaten, Spätmadrigalen, in Kanzonetten, Arien, Kantaten, "Balletti", "Ritornelli" (Cecchini, Jelić, Komnen); die Musik der Vorklassik und des Klassizismus bringt Symphonien (L. Sorkočević, A. Sorkočević, J. Bajamonti, A. Ivančić, G. M. Stratico), Sonaten und Konzerte für Violine (S. N. Spadina, G. M. Stratico, I. Jarnovic), kammermusikalische Formen wie beispielsweise Duos, Trois, Quartette (I. Jarnović, A. Sorkočević, J. Bajamonti, G. M. Stratico), Klaviermusik (L. Ebner, A. Sorkočević, T. Resti) sowie Anfänge der Vokalmusik (J. Pucić-Sorkočević); die Romantik kommt u. a. in den Anfängen mit Innovationen wie die Klavierminiatur und das romantische Lied (F. Livadić und V. Lisinski) und die Oper (V. Lisinski), um gegen Ende mit der heroischen Oper (I. Zajc), mit dem Verismus in der Oper (B. Bersa) und mit der Operette (I. Zajc, S. Albini) fortzuset- 
zen; das 20. Jahrhundert im vollen Aufschwung des Stilpluralismus - von den Elementen des Impressionismus und Expressionismus (B. Bersa, B. Kunc, I. Parać) über die kraftvolle neonationale Richtung und mit der Neoromantik (D. Pejačević, A. Dobronić, J. Gotovac, F. Lhotka, B. Baranović, S. Šulek, I. Brkanović, R. Matz u. a.) bis hin zu den Ausläufern der Darmstädter Avantgarde, des Minimalismus und der sogenannten Postmoderne (M. Kelemen, I. Malec, D. Detoni, I. Kuljerić, S. Horvat, R. Radica, S. Foretić, D. Kempf, M. Ruždjak, F. Parać, Z. Juranić und viele andere jüngere Komponisten) manifestiert hier der ganze Reichtum an Formen und Erscheinungen, den diese Musik mit den meisten europäischen Nationalkulturen und transnationalen Stilrichtungen gemeinsam teilt. Individualpsychologische und soziale Funktionen erfüllte die profane kroatische Kunstmusik im Einklang mit westeuropäischen Kontexte, die verständlicherweise durch lokale Zustände modifiziert worden sind: dalmatinische und nordadriatische Stadtkommunen (beispielsweise Cres, Rijeka, Zadar, Šibenik, Trogir, Split, Hvar u. a.) stellen jede für sich kleine, in sich geschlossene Welten mit dem vorwiegend profanen Hausmusizieren in aristokratischen und bürgerlichen Häusern vom Spätmittelalter bis zur Blütezeit der bürgerlichen Gesellschaft Ende des 19. und Anfang des 20. Jahrhunderts dar, gefolgt allerdings auch von einer sakralen Funktionalisierung der Musik in Pfarrkirchen und Domen sowie in Klöstern der Franziskaner, der Dominikaner und später auch der Jesuiten; Dubrovnik als ein Sonderfall für sich pflegt nebst allem, was von anderen dalmatinischen Städten gesagt wurde, in einer Kontinuität von über einem halben Millenium (1301-1808) auch die sogenannte Fürstenmusik (Banda di Principe; Orchestra Rettorale usw.) als einen bezahlten Ensemble, der die Bedürfnisse des Stadtstaates von der Signalfunktion bis hin zur staatlichen Repräsentanz befriedigen wird. Die Pflege der höfischen Musik wird im feudalen kroatischen Binnenland hingegen infolge konkreter Umstände der Türkenkriege erst nach der Renaissance einsetzen, und zwar an den Höfen der Patrizierfamilien Zrinski und Frankapan im 17., dann an den Höfen des kroatischen, ungarischen und österreichischen Adels (Familien Patačić, Drašković, Erdödy, Prandau, Pejačević usw.) Ende des 18. und während des 19. Jahrhunderts.

Die Komponente der städtischen und bürgerlichen Musikkultur wird sich langsam und allmählich etwa seit dem 14. Jahrhundert in den Städten wie Dubrovnik, Zagreb, Split und Zadar, etwas später dann auch in Rijeka, Osijek und Varaždin usw. entwickeln; infolge ihrer relativen Schwäche wird sie jedoch im Schatten der aristokratischen Komponente bleiben. Diese Städte wie übrigens auch die kroatische Länder als Ganzes entwickeln sich weiter, und ihre Kultur einschließlich des Musiklebens ist auf Grund ihrer Intensität und manchmal auch Qualität im Einklang mit städtischen Kulturen größerer gesellschaftlich-politischer Gebilde, denen sie verwaltungsmäßig untergeordnet sind. Diese Situation wird sich mit der Stärkung der romantisch inspirierten nationalen Bewegung zu Beginn des zweiten Drittels des 19. Jahrhunderts merklich verändern, wodurch es möglich sein wird, daß Anfang des 20. Jahrhunderts die meisten der größeren Städte ihr musikalisches Leben um Institutionen fokussieren wird: Opernhäuser mit ihren Gebäuden und ihrem professionellen Betrieb (Zagreb, Rijeka, Split, Osijek), Symphonieorchester (Zagreb, zeitweise Split, Osijek, Rijeka und Dubrovnik), Kammermusikensembles, spezialisierte Musikschulen usw. 
Ebenfalls ist es interessant, daß während einer sehr langen Periode - seit Mitte des 12. Jahrhunderts bis hin zum Abgang des feudalen "ancien régime" Mitte des 19. Jahrhunderts - wirkten in kroatischen und anderen westeuropäischen Ländern etwa dreißig Denker und Musikschriftsteller kroatischer Herkunft, die im Hinblick auf verschiedene Aspekte der Musik wertvolle Texte oder Fragmente veröffentlichten. Es waren dies mittelalterliche Gelehrte wie Herman Dalmatin und Petar Pavao Vergerije der Ältere; Humanisten, Philosophen, Theologen, Wissenschaftler und Reisebeschreiber aus der Zeit der Renaissance wie F. Grisogono-Bartolačić, B. Đurđević, L. Bassani, M. Vlačić Illyricus, P. Skalić, F. Patritius, N. V. Gučetić, M. Monaldi und F. Vrančić; Lexikographen, Ärzte, Musiktheoretiker und Geschichtsschreiber aus der Barockzeit wie J. Alberti, J. Mikalja, J. Habdelić, K. Ivanović. G. Baglivi, A. Della Bella, I. Belostenec und A. Jambrešić; Lehrbuchschreiber, Wissenschaftler, Polihistoren, Historiker und Lexikographen der klassizistischen Periode wie M. Šilobod-Bolšić, P. Serra, J. F. Domin, J. Bajamonti, J. Stulli, J. Voltiggi, T. Mikloušić u. a. Die Weichen für eine moderne Musikwissenschaft werden bereits in der zweiten Hälfte des 19. Jahrhunderts von F. Ks. Kuhač und Vjenceslav Novak gestellt; im 20. Jahrhundert werden diese Bemühungen auch von B. Širola, D. Plamenac, J. Andreis, Lovro Županović sowie von einer ganzen Reihe der auch heute noch wirkenden Musikwissenschaftler fortgesetzt. Themen, die im Mittelpunkt der älteren kroatischen Musikbetrachtung während ihrer langen, mehr als acht Jahrhunderte dauernden Geschichte waren sehr verschiedenartig: Musik und Kosmologie, Numerologie, Pädagogik, Völkerkunde, Enzyklopädistik, Poetik, Ästhetik, Lexikographie, Medizin, Akustik, Geschichtsschreibung usw. Die neuere kroatische Musikwissenschaft ist nach Definitionen ihrer thematischen Sachverhalte ein Teil der westeuropäischen Musikwissenschaft (Ethnomusikologie, historische und systematische Musikwissenschaft, Ästhetik und Soziologie der Musik usw.); ein Teil der kroatischen Musikwissensschaftler des 19. und 20. Jahrhunderts genoß die Aus- und Weiterbildung im Westen (in Frankreich, Deutschland, Österreich, England, in den USA usw.).

Andererseits ist es merkwürdig, wie geringfügig die sogenannten östlichen Themen und Inhalte die Aufmerksamkeit der kroatischen Musikkultur während ihrer dokumentierten Geschichte anzuziehen vermochten. Sie lassen sich auf wenige sporadische Fälle zurückführen: lapidare Berichte der ehemaligen türkisch-otomanischen Gefangenen B. Đurđević und L. Bassani im 16. Jahrhundert; tendenziöses Interessse des panslawischen und uniatenfreundlichen Schwärmers J. Križanić im 17. Jahrhundert; einige schriftliche Aufzeichnungen türkischer Melodien aus Bosnien aus der Feder des dalmatinischen Enzyklopädisten J. Bajamonti gegen Ende des 18. Jahrhunderts; der romantische Hang zur orientalisch anmutenden Exotik bei Ivan Zajc in manchen seiner Operetten und Opern; kurzlebige romantische Schwärmereien des prorussischen Panslawismus - beides im 19. Jahrhundert.

Was der mitteleuropäische Raum auf dem Gebiet der Kultur und Musik bedeutet, haben nicht zuletzt auch viele Referenten bei diesem Symposium zu erläutern versucht. Wie dem auch sei, es bleibt nach wie vor schwer, den Kulturbegriff und die Realität namens "mitteleuropäischer Raum" als eine bestimmte räumliche Entität zu definieren, wenngleich viele unter uns intuitiv zu ahnen und empirisch zu spüren 
geneigt sind, daß es sich hier keineswegs um eine reine Fiktion handelt. Gemeint ist darunter ein Umfeld der Gravitationspunkte in der geistigen Geographie Westeuropas; bestimmbar sind diese Punkte soziologisch und kulturologisch als deren Übergangsgebiet Osteuropa gegenüber. Für diejenige Forscher in Kroatien, die darum bemüht sind, den Habitus der kroatischen Musikkultur versuchsweise zu bestimmen, ist dieser Raum allenfalls plurizentrisch. Halbwegs poetisch gesagt, wird dieser Begriff in Kroatien mehr als eine aus vielen Sternen zusammengesetzte Galaxie und weniger als ein in sich geschlossenes, von einer einzigen Sonne abhängiges Planetensystem geahnt und gespürt. Zweifelsohne werden wir alles, was in der Musikkultur irgendwie mit Graz, Salzburg, Wien und Österreich, mit Budapest, Pécs und Ungarn, mit Mannheim, Dresden, München und Süddeutschland, mit Prag, Brünn und Tschechien, mit Bratislava, Trnava und der Slowakei, mit Warschau, Krakau und Polen, mit Ljubljana, Maribor und Slowenien, mit Zagreb und Nordwestkroatien, aber auch mit einigen norditalienischen Provinzen wie Veneto (ohne Venedig) und Furlanien mit Udine und Triest zusammenhängt, - all das werden wir als eine Art Bindeglied, als geistige Verwandtschaft und Grundlage gemeinsamer Interessen, als Beeinflussungssphäre, schöpferisches Klima und/oder als methodologische Modelle empfinden - als etwas nämlich, was uns durch konkrete und immanente Zusammenhänge äußerst vielfältig miteinander verbindet. Religiöse Aspekte sind/waren dabei überaus wichtig: explizite als Zugehörigkeit zur transnationalen Katholizität oder im weitaus geringeren Umfang zum Protestantismus; implizite als Widerspiegelung von Themen, Problemen und Lösungen auf wesentlichen existentiellen Ebenen unserer alltäglichen Lebensführungen an sich. Es ist an dieser Stelle weder möglich noch erforderlich, eine enorme Anzahl von Komponisten, Reproduktivkünstlern und Musiktheoretikern zu nennen, die, aus kroatischen Landen abstammend, auf Grund ihres freien Willens einen oder mehrere Mittelpunkte des Musiklebens Mitteleuropas als ihre eigene Ausbildungsstätte bzw. als Quelle ihrer dauerhaften schöpferischen Inspiration gewählt haben. Stilmerkmale, Ideen, Wissensprofile, Arbeitsmethoden, Organisierungsmodalitäten - all das sind Ausprägungen des Bewußtseinszustands und nicht selten auch die Beweggründe zum Wirken dieser kroatischen Künstler und Wissenschaftler auf dem Gebiet der Musik, mehr oder minder jedoch auch im Rahmen aller anderen Künste: der Malerei, der Architektur, der Literatur usw.

Das zentrale Problem hinsichtlich der Identität der kroatischen Musikkultur im engeren und der allgemeinen Kultur im weiteren Sinn des Wortes ist keine künstliche Frage, die sich durch die Logik der sogenannten hohen Politik, der Medien und der Linguistik in den letzten acht Jahrzehnten aufdrängt - ob Kroatien nämlich zu Mitteleuropa oder zum europäischen Osten gehörig sei. Eine so gestellte Frage mindestens im Hinblick auf Kultur und Kunst, insbesondere aber in bezug auf die Musik - kann lediglich einen hohen Grad der durch nichts zu rechtfertigenden Ignoranz oder eine ethisch dubiose und tendenziöse Neigung zur Fälschung der historischen wie auch der gegenwärtig aktuellen Wirklichkeit darstellen. Die richtige, mit dem Problem dieser Identiät tatsächlich zusammenhängende Frage läuft darauf hinaus, in welchem Maße die kroatische Kultur, Kunst und Musik durch den Dualismus zweier sich gänzlich voneinander abhebender Pole - des Mediterranen und des Mit- 
teleuropäischen - gespalten sind? Das Problem besteht also in einer (geographisch gesehen) vertikalen (Nord-Süd) imaginären Einteilung; es ist dies eine Einteilung zwischen dem musikalisch mitteleuropäischen und dem mediterranen Kroatien; gar keine Rede von einem Kroatien im musikalischen Westen oder Osten Europas (horizontale Einteilung Ost-West).

Bei einer solchen Problemartikulierung interessieren uns vor allem die Einflußsphären des musikalischen Nordens und Südens, zwei Kreise, die sich in der geistigen Geometrie überlappen; es interessiert uns ihre osmotische Abgrenzung in Kroatien, Kräfte, Domänen, Verwandlungen ihrer Einflüsse in der durchlebten historischen Dynamik und Wandelbarkeit. Dasjenige freilich, womit wir uns heutzutage beim Reflektieren der kroatischen Kunstmusik und Kultur befassen bzw. befassen sollten, wenn es darum geht, den Geist ihres Wesens nachzuvollziehen, ist folgendes: die Frage nach der Reichweite von Einflüssen aus Aquilea, Salzburg und Kalocsa bzw. Györ auf die kroatische Musik im Mittelalter sowie die Reminiszenzen des beneventanischen Komplexes in Dalmatien; die Frage nach der teilnehmenden Anwesenheit kroatischer Musiker und Künstler am humanistisch bedeutungsvollen Hof des Königs Matthias Corvinus Ende des 15. Jahrhunderts; Fragen der mißlungenen Reformation überall in Kroatien, von Međimurje und Istrien bis nach Split sowie der Gegenreformation, bzw. der katholischen Erneuerung im 17. und zum Teil im 18. Jahrhundert im kroatischen Binnenland; die Frage nach dem Durchbruch der Vorklassik und Klassik, der postklassizistisch/vorromantischen Musik in Dubrovnik und Dalmatien vor und nach dem Fall Venetiens; die Frage nach der Ursache und dem Verhältnis von musikalischen Einflüssen aus Venetien, der Lombardei, Rom und Neapel in Richtung Dalmatien und Dubrovnik im selben Zeitalter; das Problem des Nationalen in der Musik Lisinskis und Zajcs sowie des Nationalistischen in Kuhačs Musikbetrachtungen im 19. Jh.; Erneuerung der Bedeutung der Moderne in der kroatischen Musik als Gegenpol zum naiven Nationalismus um die Jahrhundertwende; eine reife neonationale Richtung, das Verhältnis zur zweiten Wiener Schule und der erste künstlerisch-ideelle Widerstand bei der mißlungenen Begegnung mit dem europäischen Osten im ersten Jugoslawien; der Konflikt zwischen dem Traditionalismus und Modernismus der 60er Jahre des 20. Jahrhunderts und die Rolle der Zagreber Musikbiennale bei der Monopolsprengung der realsozialistischen kunstideologischen Diktatur; das einsetzende Ende des Modernismus und der Auftritt der Postmoderne mit dem zähflüssigen Zerfall des sozialistischen Jugoslawiens; künstlerische Konfusion der Nachkriegstraumata und der schöpferischen Freiheit im unabhängigen Kroatien nach 1991 usw.

Kehren wir doch zum Schluß wieder zu dem zurück, womit wir begonnen haben, und das ist die fundamentale Frage nach dem Verhältnis der kroatischen Kunstmusik zum mitteleuropäischen Raum. Meine Antwort würde hierzu folgendermaßen lauten: die kroatische Kunstmusik und die Musikkultur lassen sich aus der kroatischen Perspektive in einem gewichtigen Teil als ein Bestandteil des mitteleuropäischen Raumes wahrnehmen. Dieser Raum aber, der in sich keineswegs einheitlich, sondern auf eine Reihe partikulärer Interessensphären unterschiedlicher Größe und Stärke dispersiert ist, nimmt die kroatische Musikkultur als seinen Be- 
standteil sehr selten wahr; in Übersichten, Abhandlungen, Klassifizierungen und Auffassungen wird sie häufiger als eine abgesonderte Entität mit eventuell vorkommenden Elementen mitteleuropäischer Provenienz behandelt. Dabei macht er am häufigsten Fehler, indem er bewußt oder unbewußt der sogenannten hohen Politik Folge leistet, die auch heutzutage viel zu oft Kroatien im Osten Europas ansiedelt, wobei er, von der mitteleuropäischen Komponente einmal abgesehen, dessen andere, gleichermaßen wichtige mediterrane Komponente völlig außer Acht läßt.

Die kroatische Darstellung der musikalischen Faktographie und die Interpretation des eigenen musikalischen Habitus kann keineswegs den Diskurs über den Dualismus ihres eigenen mitteleuropäisch-mediterranen Charakters und der damit verknüpften Identität vermeiden; freilich darf sie auf der Aufhebung dieser faktographisch unbegründeten und daher trügerischen Wahrnehmung der kroatischen Musikkultur und Kunstmusik im ganzen - in Mitteleuropa und insbesondere in seinem Westen - als eines überwiegenden oder sogar integralen Teils des europäischen Ostens unermüdlich bemüht sein. Kroatien befindet sich am Rande des mittleren und darüber hinaus des westlichen Europas; selbstverständlich ist es ein Übergangsland und eine Übergangskultur in Richtung Osten, aber Kroatien ist unbestrittenerweise auch ein Land aus dem westslawischen und dadurch mitteleuropäischen musikalisch-kulturellen Umkreis. Einige neuere Indizien (Neuausgaben der Enzyklopädien wie Grove, MGG, Larousse; Zusammenarbeit mit der Fondazione Levi in Venedig, Mitarbeit am Österreichischen biographischen Lexikon, gewisse diskographische Interesse für kroatische Kunstmusik in Österreich für D. Pejačević und Deutschland [für Familie Sorkočević] usw.) zeigen indessen, daß der Prozeß einer immer größer werdenden Beteiligung Kroatiens an derzeit aktuellen kulturellen, gesellschftlichen und politischen Umstrukturierungen und Integrationen in Europa einem anderen Prozeß der eigenartigen musikalisch-kulturologischen Bewußtmachung überaus behilflich zur Seite steht. Es fragt sich deshalb, ob ein Land, dessen Melodie der plebiszitär bestimmten Staatshymne donizettischer Provenienz ist und deren erster Vers, mit dem Eigenschaftswort "schön" beginnend, die zentrale Kategorie der westlichen musikalischen Ästhetik von Plato bis Hanslick inauguriert, - ob ein solches Land mehr als symbolisch verdient, die nach wie vor bestehenden und hier nur knapp formulierten Zusammenhänge von nun an in einem neuen, mit der historischen Wahrheit in Einklang gebrachten Licht zu betrachten?

\section{Literatur (Auswahl)}

Andreis, Josip: Music in Croatia, Zagreb 1982, 2. Aufl.

Barbarić, Damir \& Benedikt, Michael: Ambivalenz des Fin de siècle Wien-Zagreb, Böhlau Verlag, Wien-Köln-Weimar 1998.

Blažeković, Zdravko: Catalogues of Music Manuscripts and Prints in the Historical Archives and the Museum of the City of Dubrovnik, JAZU, Zagreb 1988.

Breko, Hana: Misal MR 70 zagrebačke Metropolitanske knjižnice, HMD, Zagreb 2003.

Cavallini, Ivano: I due volti di Nettuno. Studi su teatro e musica a Venezia e in Dalmazia dal Cinquecento al Settecento, Libreria Musicale Italiana Editrice, Lucca 1994. 
MUZIKOLOŠKI ZBORNIK • MUSICOLOGICAL ANNUAL XL

Dadić, Žarko: Povijest egzaktnih znanosti u Hrvata, Vol. 1 \& 2, SNL, Zagreb 1982.

Demović, Miho: Musik und Musiker in der Republik Dubrovnik (Ragusa) vom Anfang des XI. Jh. bis zur Mitte des XVII. Jh., Regensburg 1981.

Demović, Miho: Glazba i glazbenici u Dubrovačkoj Republici od polovine XVII. do prog desetljeća XIX. stoljeća, JAZU, Zagreb 1989.

Demović, Miho (Hrsg): Zagrebački sekvencijar, Zagreb 1994.

Demović, Miho (Hrsg): Trogirski evandelistar, Split 1997.

Enix, Margery: Rudolf Matz. Cellist, Teacher, Composer, Dominis Publishing, Ottawa 1996.

Golub, Ivan (Hrsg.): Hrvatska i Europa. Kultura, znanost $i$ umjetnost, Vol. 3: Barok i prosvjetiteljstvo (XVIII-XVIII. stoljeće), Školska knjiga, Zagreb 2003.

Grgić, Miljenko: Glazbena kultura u splitskoj katedrali 1750-1940, HMD, Zagreb 1997.

Hercigonja, Eduard (Hrsg.): Hrvatska i Europa. Kultura, znanost i umjetnost, Vol. 2: Srednji vijek i renesansa (XIII-XVI. stoljeće), Školska knjiga, Zagreb 2000.

Juričić, Vedrana: Guide through Music Libraries and Collections in Zagreb, HMDHBD-HAZU, Zagreb 1997.

Juričić, Vedrana: Catalogue of Music Manuscripts and Prints in the Benedictine Convent of St. Peter in Cres, HMD, Zagreb 2000.

Katalinić, Vjera: Glazbeni arhiv samostana Male braće u Dubrovniku. Rani rukopisi od početka 18. stoljeća do oko 1820, Samostan Male braće u Dubrovniku, KS-SMB, Zagreb-Dubrovnik 1985, 623-664.

Katalinić, Vjera: Catalogue of Music Manuscripts and Prints in the Franciscan Monastery in Omiš, HAZU, Zagreb 1991.

Katalinić Vjera (Hrsg.): Ferdo Wiesner Livadić. Život i djelo ( Life and Opus, HMD, Zagreb 2003.

Katalinić, Vjera \& Tuksar, Stanislav (Hrsg.): Mladi Zajc / Young Zajc: Beč / Vienna (1862-1870), Izdavački centar Rijeka, Rijeka 2003.

Katalinić, Vjera \& Tuksar, Stanislav (Hrsg.): Musical Cultures in the Adriatic Region During the Age of Classicism, HMD, Zagreb 2004.

Kos, Koraljka: Musikinstrumente im mittelalterlichen Kroatien, Muzikološki zavod MA, Zagreb 1972.

Kos, Koraljka: Luka Sorkočević i njegov doprinos pretklasičnoj instrumentalnoj muzici. Bilješke o kompozicijskom slogu i prilog stilskoj analizi njegova djela, Arti musices, 5 (1974), 45-89.

Kos, Koraljka: Geschichte, Stand und Perspektiven der Forschungen zur mittelalterlichen Kirchenmusik in Kroatien, International Review of the Aesthetics and Sociology of Music, VIII/2 (1975): 289-307.

Kos, Koraljka: Style and Social Background of Croatian Renaissance Music, Arti musices, sp. issue 2 (1979), 45-89.

Kos, Koraljka: Dora Pejačević. Leben und Werk, MIC, Zagreb 1987.

Kos, Koraljka - Šojat, Antun - Zagorac, Vladimir: Pavlinski zbornik 1644. Transkripcija $i$ komentari, Vol. I \& II, HAZU, Zagreb 1991.

Krpan, Erika (Hrsg.): The Zagreb Music Biennale 1961-2001, Croatian Composers' Society, Zagreb 2000. 
MUZIKOLOŠKI ZBORNIK • MUSICOLOGICAL ANNUAL XL

Majer-Bobetko, Sanja: Estetika glazbe u Hrvatskoj u 19. stoljeću, JAZU, Zagreb 1979.

Miklaušić-Ćeran, Snježana: Glazbeni život Zagreba u XIX. stoljeću, HMD, Zagreb 2001.

Stipčević, Ennio (Hrsg): Glazbeni barok u Hrvatskoj, OGV, Osor 1989.

Supičić, Ivo (Hrsg.): Croatia in the Early Middle Ages. A Cultural Survey, Philip Wilson Publishers, London 1999.

Šaban, Ladislav: 150 godina Hrvatskog glazbenog zavoda, HGZ, Zagreb 1982.

Tuksar, Stanislav: Croatian Renaissance Music Theorists, MIC, Zagreb 1980.

Tuksar, Stanislav (Hrsg.): Luka i Antun Sorkočević, MIC-OGV, Zagreb-Osor, 1983.

Tuksar, Stanislav (Hrsg.): Ivan Mane Jarnović - hrvatski skladatelj, I \& II, OGV-MIC, Zagreb-Osor 1978 \& 1980.

Tuksar, Stanislav: New Musical Sources in Croatia (Yugoslavia), Acta Musicologica, LVII/I (1985): 121-138.

Tuksar, Stanislav: Glazbeni arhiv samostana Male braće u Dubrovniku. Opis fonda i rane tiskovine, Samostan Male braće u Dubrovniku, KS-SMB, Zagreb-Dubrovnik 1985, 665-773.

Tuksar, Stanislav: Imprimés musicaux européens anciens et rares dans les archives croates, u: Les Croates et la civilisation du livre, Croatica Parisiensia (Paris), Presses de l'Université de Paris-Sorbonne, 1 (1986): 67-74.

Tuksar, Stanislav: Catalogue of Music Manuscripts and Prints in the City Museum of Split, JAZU, Zagreb 1989.

Tuksar, Stanislav: Hrvatska glazbena terminologija u razdoblju baroka. Nazivlje glazbala $i$ instrumentalne glazbe u hrvatskim tiskanim rječnicima od 1649. do 1742. Organografsko-povijesni i sociolingvistički aspekti, Hrvatsko muzikološko društvo - Muzički informativni centar, Zagreb, 1992.

Tuksar, Stanislav: Music Research Libraries, Archives and Collections in Croatia, International Review of the Aesthetics and Sociology of Music, 23/2 (1992): 119-140.

Tuksar, Stanislav (Hrsg.): Musical Baroque, Western Slavs, and the European Cultural Communion / Glazbeni barok $i$ zapadni Slaveni u kontekstu europskog kulturnog zajedništva, Hrvatsko muzikološko društvo-HAZU, Zagreb 1993.

Tuksar, Stanislav: Music Writers, Theorists and Lexicographers of Mediterranean Croatia in the c.1500-c.1800 Period, u: Actas del XV Congreso de la Sociedad Internacional de Musicologia, Revista de Musicologia, XVI/3 (1993): 1407-1415.

Tuksar, Stanislav: The Madrigal. Destiny of a Form in Croatian Musical Culture, u: $I l$ madrigale oltre il madrigale, Atti del IV Convegno internazionale sulla musica italiana nel secolo XVII, A.M.I.S., A. Colzani-A. Luppi-M. Padoan (Hrsg.), Como 1994, 49-58.

Tuksar, Stanislav: Music by Eighteenth-Century German and Austrian Composers Preserved in Venetian Dalmatia and Dubrovnik. Differences and Similarities, u: Relazioni musicali tra Italia e Germania nell'età barocca / Deutsch-italienische Beziehungen in der Musik des Barock, Atti del VI Convegno internazionale sulla musica italiana nei secoli XVII-XVIII, A.M.I.S., Loveno di Menaggio (Como), Como 1997, 447-461. 


\section{MUZIKOLOŠKI ZBORNIK • MUSICOLOGICAL ANNUAL XL}

Tuksar, Stanislav (Hrsg.): Zagreb 1094-1994. Zagreb i hrvatske zemlje kao most izmedu srednjoeuropskih i mediteranskih glazbenih kultura / Zagreb and Croatian Lands as a Bridge Between Central-European and Mediterranean Musical Cultures, HMD-HAZU, Zagreb 1998.

Tuksar, Stanislav (Hrsg.): Srednjovjekovne glazbene kulture na istočnoj i zapadnoj obali Jadrana do početka 15. stoljeća / Mediaeval Musical Cultures on the Eastern and Western Adriatic Shores Until the Beginning of the 15th Century, HMD, Zagreb 2000.

Tuksar, Stanislav (Hrsg.): Muzička akademija / Academy of Music 1922-2002, Muzička akademija, Zagreb 2002.

Županović, Lovro: Centuries of Croatian Music, Vol. I \& II, MIC, Zagreb 1984, 1987. 